\title{
Imagination, représentation et impression : Quelques remarques grammaticales de Wittgenstein
}

\author{
Par CHARLOTTE GAUVRY \\ FNRS, Université de Liège
}

Résumé Notre article explore les analyses de Wittgenstein développées dans ses Remarques sur la philosophie de la psychologie II de 1948 qui examinent la corrélation entre la représentation et l'imagination. Ces remarques ont une portée essentiellement grammaticale en ceci qu'elles ont pour finalité de clarifier la manière dont les concepts de « représentation », d' « image » et d' « impression sensorielle» sont effectivement mobilisés dans nos jeux de langage ordinaires. Le premier objectif de l'article est de souligner la dimension critique de l'analyse de Wittgenstein en manifestant sa portée polémique à l'encontre de la conception de Hume qui associe l'imagination à une « idée » ou « image interne » et qui postule la thèse selon laquelle l'image interne et l'impression sensorielle diffèrent par leur degré de vivacité. L'un des enjeux de l'analyse wittgensteinienne est en effet de montrer qu'une telle conception repose sur un «mythe de l'image interne ». La preuve en est qu'il est possible de dissocier le concept d'imagination (entendue comme représentation), de ceux d'image et d'impression, même si ces concepts sont souvent corrélés. Plus positivement, le deuxième objectif de l'article est de souligner quelques spécificités grammaticales de la représentation par image. L'analyse conceptuelle manifeste en effet le fait que seule l'imagination, entendue comme activité de représentation, à la différence de l'impression, 1/ est soumise à la volonté, 2/ est une pratique normée, 3/ présente une dimension créatrice. En raison de ces spécificités, il s'avère que seule l'imagination est susceptible d'inventer de nouveaux jeux de langage. En conséquence, l'article insiste pour conclure sur le fait que l'imagination se présente comme un outil méthodologique privilégié de l'analyse wittgensteinienne dont l'une des aspirations ultimes est d'obtenir une visée synoptique de nos différents jeux de langage. 


\section{Introduction}

Dans différents documents dactylographiés qui datent de l'année 1948, Ludwig Wittgenstein développe une longue série de remarques grammaticales sur la «Vorstellungskraft », à savoir sur la faculté de "représentation », et sur ses liens avec l' «imagination». L'analyse a été reproduite dans ses Remarques sur la philosophie de la psychologie $I^{1}$ et, en partie, dans ses Fiches $^{2}$ qui datent de la même époque. La méthode à laquelle a recours Wittgenstein pour caractériser l'imagination s'apparente à celle de la "psychologie descriptive », du moins au sens où l'expression en a réellement un pour Wittgenstein. Le philosophe propose en effet une série d'analyses descriptives des jeux de langage qui mettent en jeu des concepts psychologiques, en l'occurrence ceux d'image, d'impression sensorielle et de représentation.

Notons que dans ces remarques, l'analyse porte davantage sur la grammaire de la «représentation» (Vorstellung) ou du « représenter » (vorstellen) (que les traducteurs anglais ${ }^{3}$ traduisent par « image », « imaging » et «imagine »), plutôt que sur celles de l'«image» (Bild) ou même du « s'imaginer » (sich einbilden) à proprement parler. Il est cependant parfois question d' « image de la représentation ${ }^{4}$ (Vorstellungs-bild). Ce choix terminologique n'est pas fortuit. Ainsi que nous le montrerons, il a pour principale fonction de contourner le lexique des théories de "l'image interne » (das innere Bild), ou du moins de libérer l'analyse de l'imagination de certains présupposés hérités des théories modernes du XVII ${ }^{\mathrm{e}}$ siècle.

Il convient dès lors de prendre la mesure de la dimension polémique des remarques de Wittgenstein et de caractériser plus avant l'une de ses cibles : la conception humienne des «idées » entendues comme images mentales de nos impressions sensorielles. Par le moyen de jeux de comparaison d'une grande diversité d'usages, Wittgenstein entend en effet clarifier les jeux de langage qui mobilisent les concepts de représentation et d'impression et manifester à nouveaux frais leurs relations complexes avec le concept

1 L. Wittgenstein, Bemerkungen über die Philosophie der Psychologie II (1948), G.E.M. Anscombe et G.H von Wright (éds.), Oxford, Blackwell, 1980, tr. fr. G. Granel, Remarques sur la philosophie de la psychologie II (RPP II par la suite), Mauvezin, TER, 1994, notamment $\S \S 63-147$.

2 L. Wittgenstein, Zettel (1945-1948), G.E.M. Anscombe et G.H. vo Wright (éds.), tr. fr. J.-P. Cometti et É. Rigal, Fiches (F par la suite), Paris, Gallimard, 2008.

3 Ces traducteurs sont en l'occurrence C.G. Luckhardt et M.A.E. Aue dans L. Wittgenstein Remarks on the Philosophy of Psychology, Oxford, Blackwell, 1998. 4 Voir par ex. RPPII § 69, p. 15. 
d' « image ». Ainsi qu'il le remarque par exemple dans le paragraphe 112 des Remarques sur la philosophie de la psychologie:

$\mathrm{Ni}$ «représentation » [Vorstellung] ni “impression" [Eindruck] ne sont des concepts de l'ordre de l'image [Bild], bien que dans les deux cas une corrélation avec une image $[$ Bild $]$ ait lieu, différente dans l'un et dans l'autre ${ }^{1}$.

L'enjeu des analyses grammaticales de Wittgenstein est précisément de parvenir à clarifier cette corrélation et de montrer ce faisant, à l'encontre des idées préconçues, que l'imagination n'est ni une pure activité mentale déconnectée des impressions sensorielles, ni un simple prolongement de la perception. Grâce aux outils de la philosophie du langage ordinaire, à savoir de l'analyse grammaticale des jeux de langage, Wittgenstein entend ainsi repenser l'articulation entre impression sensorielle, image et représentation.

\section{Une critique des images internes}

Si Wittgenstein ne cite pas systématiquement ses sources, il est manifeste que les analyses grammaticales de Wittgenstein sur l'imagination, du moins celles des Remarques qui nous intéressent, ont une portée polémique à l'encontre de la conception psychologique des «idées » ou images mentales des Modernes, de Hume en particulier. Bon nombre des concepts et arguments mobilisés lui sont en effet empruntés.

À titre de préambule, il n'est en conséquence pas faux de dire ${ }^{2}$ que Wittgenstein souscrirait pour une bonne part au diagnostic établi par Sartre au début de son essai de psychologie phénoménologique de 1940 qu'est $L^{\prime}$ 'imaginaire ${ }^{3}$. D'après ce diagnostic, il existe une conception du sens commun de l'imagination, qui est partagée par la plupart des philosophes et des psychologues de la fin du XIX ${ }^{\mathrm{e}}$ et du début du $\mathrm{XX}^{\mathrm{e}}$ siècles qui remonte aux empiristes britanniques. Plus exactement, comme l'affirme explicitement Sartre, elle «trouve chez Hume son expression la plus claire » ${ }^{4}$. Selon cette conception dominante, l'imagination se caractérise par une double croyance d'après laquelle l'image est «dans la conscience et l'objet de l'image est

1 RPP II § 112, p. 24.

2 Voir par exemple H.-G. Glock, A Wittgenstein Dictionary, Oxford, Blackwell, 1996, section « imagination ».

3 J.-P. Sartre, L'imaginaire, Paris, Gallimard, 1940.

4 Ibid., p. 16. 
dans l'image $»^{1}$. C'est ce que Sartre caractérise comme une «double erreur » qu'il fustige sous le terme générique d' «illusion d'immanence». Pour reprendre son exemple, selon cette conception, dire que «"j'ai une image" de Pierre $»^{2}$, cela consiste à « pense[r] présentement que j'ai un certain portrait de Pierre dans la conscience » et que ce qui est ainsi « contenu » dans ma conscience, c'est ce portrait de Pierre - cette image de Pierre - et non pas Pierre lui-même, «l'homme de chair et d'os » qui « ne serait atteint que très indirectement», d'une façon «extrinsèque », dans la faculté d'imagination. L'image serait comme un « contenu de conscience », interne à la conscience ${ }^{3}$. Or Sartre et Wittgenstein ont en commun de considérer que la théorie de Hume préfigure à bien des égards cette conception qui reste dominante au $\mathrm{XX}^{\mathrm{e}}$ siècle. Il convient dès lors d'en rappeler les principes pour comprendre le sens des déplacements opérés ensuite par Wittgenstein.

Rappelons qu'au tout début du Traité de la nature humaine ${ }^{4}$, dans la section consacrée à " l'origine des idées », Hume affirme qu'il existe deux genres de " perceptions de l'esprit humain », les «impressions» et les " idées » ${ }^{5}$, qui ne diffèrent que par leur degré de vivacité. Hume précise en effet que les « idées », qui se caractérisent par leur moindre degré de vivacité, doivent être considérées comme "les images affaiblies [des impressions] dans la pensée et le raisonnement $\gg{ }^{6}$. Comme le notera Sartre ultérieurement en relisant ce passage : "Ces idées ne sont autres que ce que nous nommons images $\gg^{7}$. Qui plus est, Hume ajoute que ces idées se distinguent ellesmêmes les unes des autres en fonction de leur degré de vivacité et de fidélité aux impressions, selon qu'il s'agisse d'idées de la mémoire ou d'idées de l'imagination à proprement parler. Les images (ou idées) de l'imagination se caractérisent en l'occurrence par leur moins grande intensité et par leur plus grand degré de liberté à l'égard des impressions dont elles sont issues :

1 Ibid., p. 15.

2 Pour cette citation et les suivantes, ibid. p. 17.

3 En un sens, la critique est largement préfigurée par la critique plus générique de l'« image interne » de la première Recherche logique de Husserl. Voir E. Husserl, Recherche logique 1. T. II/vol. $1:$ Recherches pour la phénoménologie et la théorie de la connaissance, Paris, PUF, 1961, §§ 17-19.

4 D. Hume, Traité de la nature humaine, tr. fr. Ph. Baranger et Ph. Saltel, Paris, Flammarion, 1995.

5 Ibid., p. 41

6 Ibidem.

7 J.P. Sartre, L'imaginaire, op. cit., p. 16. 
À première vue, il est évident, que les idées de la mémoire sont beaucoup plus vives et fortes que celles de l'imagination et que la première de ces facultés peint ses objets avec des couleurs plus franches que celles qui sont utilisées par la seconde ${ }^{1}$.

L'imagination n'est pas tenue de respecter identiquement l'ordre et la forme des impressions originelles, tandis que sur ce point, la mémoire est, d'une certaine manière, assujettie, sans aucun pouvoir de modification ${ }^{2}$.

De ce bref rappel, il ressort que la conception empiriste de Hume consiste à soutenir la thèse selon laquelle l'imagination est une faculté psychique que se caractérise avant tout par son contenu mental, à savoir une "idée », qui se présente comme une image d'une impression, peu intense et peu fidèle.

Or l'analyse de Wittgenstein a ceci de commun avec celle de Sartre qu'elle se méfie du concept d' «image interne» (sans nécessairement le répudier totalement pour autant) et qu'elle entend plutôt accorder toute attention à l'analyse de la pratique (de l'acte, dirait Sartre) qu'est l'imagination $^{3}$. À notre connaissance, le nom de Hume n'apparaît pas explicitement dans les Remarques sur la philosophie de la psychologie. Il n'en reste pas moins que Wittgenstein mobilise clairement le lexique humien (celui de 1' "impression» pour commencer) et examine la pertinence du principal critère que Hume mobilise pour distinguer l'«idée » de l'«impression »: celui de la «vivacité ». Dans le paragraphe 63 de ces Remarques de 1948, dans le style très laconique qui est souvent le sien, Wittgenstein répudie ainsi le critère : «Représentation auditive [Gehörsvorstellung], représentation visuelle [Gesichtsvorstellung], comment se distinguent-elles des sensations ? Pas par leur "vivacité"»". Un tel critère, selon Wittgenstein, n'est en effet pas pertinent pour distinguer la représentation, de la sensation ou de l'impression. Ainsi qu'il le remarque, il n'est pas exclu que certaines représentations soient d'une beaucoup plus grande intensité que les impressions. Qui plus est, l'éventuelle différence d'intensité entre les deux expériences n'est parfois pas manifeste. Pour ces différentes raisons, il n'est pas d'usage de distinguer dans le langage ordinaire l'une et l'autre expérience en arguant de cet argument de la « vivacité » :

1 D. Hume, Traité de la nature humaine, op. cit., p. 50.

2 Ibid., p. 51.

3 La ressemblance avec l'analyse de Sartre s'arrête, selon nous, à peu près là : Wittgenstein ne souscrit guère plus qu'au diagnostic de départ de L'imaginaire.

4 RPP II § 63, p. 14. 
$\S 98$. Si, effectivement, quelqu'un disait: «Je ne sais pas si je vois en ce moment un arbre, ou si je m'en représente [vorstellen] un », je croirais d'abord qu'il veut dire : " ou si je m'imagine [einbilden] seulement qu'il y en a un là-bas ". Si ce n'était pas cela qu'il voulait dire, je ne le comprendrais simplement pas. - Mais si quelqu'un voulait m'expliquer ce cas en disant «C'est qu'il a des représentations [Vorstellungen] si extraordinairement vivaces, qu'il est capable de les prendre pour des impressions sensorielles » - y comprendrais-je maintenant quelque chose ${ }^{1}$

Vraisemblablement pas car ce n'est précisément pas ce qu'il serait d'usage de dire. Wittgenstein est plus clair encore au paragraphe suivant :

Si quelqu'un me disait «Il arrive souvent que je ne sache pas si je vois quelque chose, ou si je ne fais que me le représenter », alors je n'appellerai pas cela un cas de super-vivacité de la représentation ${ }^{2}$.

De ces quelques exemples, on peut à tout le moins déduire que, s'il y a une différence entre la réception d'une impression sensorielle et la représentation d'une image, le critère humien de l'intensité ou de « la vivacité » n'est pas susceptible de la manifester.

Qui plus est, en amont de cette distinction, Wittgenstein se méfie de la rhétorique de 1'« image » (Bild), du moins de l'«image interne» (inneres Bild) que seul serait susceptible de voir notre « œil intérieur». Une telle conception repose selon lui sur ce qu'il nomme un «mythe de l'image interne $»^{3}$ qui n'est d'aucun usage dans le langage. Comme il le remarque en effet, "nous n'apprenons pas la signification [de concepts comme "voir»] en lien avec le concept de "voir interne" [inneres Sehen]" ${ }^{4}$. Comme l'affirmaient déjà les Recherches philosophiques qui sont un peu antérieures à ces remarques «psychologiques », quand je dis que j'imagine quelque chose de rouge, mon affirmation ne procède pas par la " reconnaissance ${ }^{5}$ d'une image mentale du «rouge»: "si tu dis que tu as vu en imagination quelque chose de rouge, il s'agira bien de quelque chose de rouge. D'habi-

1 RPP II § 98, p. 21.

2 RPP II $\S 99$, p. 22.

3 RPP II § 109, p. 23.

4 RPP II $§ 68$, p. 15.

5Voir L. Wittgenstein, Philosophische Untersuchungen/ Philosophical Investigations (Partie $1: 1945$; Partie 2 : 1947-1949), G.E.M. Anscombe et R. Rhees (éds.), tr. fr. F. Dastur, M. Elie, J-L. Gautero, D. Janicaud, É. Rigal, Recherches philosophiques (RP par suite), Paris, Gallimard, 2004, § 378, p. 172.

96

Bull. anal. phén. XIII 2 (2017)

http://popups.ulg.ac.be/1782-2041/ @ 2017 ULg BAP 
tude, tu sais bien ce qu'est le "rouge" $\gg{ }^{1}$. Car ce que je reconnais, c'est seulement la manière dont il faut employer le mot rouge, la façon « dont on peut dire à juste titre [que c'est rouge] $»^{2}$. Aussi, comme suggéré d'emblée, si Wittgenstein répudie l'idée selon laquelle l'imagination procède par la formation d'une image interne, c'est d'abord parce que son analyse descriptive se situe au niveau conceptuel. Que l'on puisse parler de ce que l'on a imaginé suggère à tout le moins que l'image en question n'est pas, du moins pas seulement, une image interne. Plus fondamentalement, comme l'affirmait déjà Wittgenstein à propos de l'utilisation des symboles mathématiques en 1939, ce qui importe, ce n'est pas l'éventuel processus interne mais plutôt la manière dont nous mobilisons nos concepts dans le langage :

§ 38. Il faut sans cesse se rappeler à nouveau le peu d'importance d'un «processus interne » ou d'un " état interne », et se demander « Pourquoi estil censé être important? En quoi me concerne-t-il » ? Ce qui est important c'est la manière dont nous utilisons les propriétés mathématiques ${ }^{3}$.

De même, ce qui intéresse Wittgenstein à la fin des années 1940, c'est moins l'analyse de nos processus psychiques et de nos (supposées) images mentales que celle de ce que j'ai dit avoir vu (ou imaginé). Comme l'affirme déjà Wittgenstein on ne peut plus clairement dans les Recherches philosophiques : « Nous n'analysons pas un phénomène (la pensée par exemple), mais un concept (celui de la pensée par exemple), et donc l'application d'un mot $»^{4}$. En l'occurrence, c'est l'usage des mots «représentation », "impression sensorielle » et " image » qu'entendent préciser les analyses des $R e$ marques sur la philosophie de la psychologie sur lesquelles nous nous appuyons.

1 RP $\S 386$, p. 174.

2 RP $\S 379$, p. 174, à propos de l'application du mot « douleur ».

3 L. Wittgenstein, Bemerkungen über die Grundlagen der Mathematik (1937-1944),

G.E.M. Anscombe, G.H. von Wright et R. Rhees (éds.), Bd 6, Francfort-sur-le-Main, Suhrkamp, 1954, trad. fr. M.-A. Lescourret, Remarques sur les fondements des mathématiques, Paris, Gallimard, 1983, § 38 .

4 RP $\S 383$, p. 173. 


\section{Une méthode de description conceptuelle}

Afin de clarifier la grammaire des jeux de langage qui mobilisent ces concepts, Wittgenstein procède, comme à l'accoutumée, par jeux de comparaisons, c'est-à-dire en comparant ce qui distingue des jeux de langage comme : «Regarde cette figure! » et «Représente-toi cette figure ! » ${ }^{1}$. En étant sensible à leurs différences, sans pour autant les accentuer, Wittgenstein entend montrer que «les jeux de langage employant ces deux concepts sont radicalement différents mais ont un rapport $»^{2}$.

Wittgenstein adopte cette méthode car il considère qu'elle seule est susceptible de clarifier les relations entre les concepts. Il émet en particulier des doutes quant à l'idée qu'une description "empirique » de deux comportements serait par exemple susceptible de clarifier la différence entre les expériences de vision et d'imagination. Il constate en effet que si « quelqu'un dit: "je vois une maison avec des volets verts" ", il n'est pas possible de répondre par simple observation en troisième personne : «Il ne les voit pas, il se les représente seulement ${ }^{3}$, comme si l'on pouvait distinguer par observation "l'air» [aussehen] qu'a quelqu'un qui voit de celui qu'a quelqu'un qui imagine seulement quelque chose. C'est la raison pour laquelle Wittgenstein, qui doute plus sérieusement encore des vertus de la méthode introspective, préconise de s'en rapporter à l'analyse des différences que manifestent nos usages des concepts. Il formule très clairement cette thèse méthodologique au paragraphe 87 :

Que sais-tu de la ressemblance de son impression visuelle [Gesichtseindrucks] et de sa représentation visuelle [Gesichtsvorstellung] ?! [...] Elle ne se manifeste que dans les expressions qu'il a tendance à utiliser ${ }^{4}$.

À titre d'illustration de sa méthode, on peut relever trois remarques grammaticales (tirées des Remarques sur la philosophie de la psychologie) que formule Wittgenstein après avoir comparé différents énoncés ordinaires :

— Première remarque : "Pendant que je regarde [sehe] un objet, je ne peux pas me le représenter [vorstellen] ${ }^{5}$. Il semble en effet que, dans la plupart des cas, il ne soit pas grammaticalement correct de dire qu'on regarde et qu'on imagine un même objet en même temps. Comme le concède

1 RPP II $\S 63$, p. 14.

$2 \mathrm{~F} \S 625$.

3 RPP II $\S 134$, p. 28.

4 RPP II $\S 87$, p. 18.

5 RPPII $\S 63$, p. 14. 
Wittgenstein, il existe cependant des contextes dans lesquels cela a vraisemblablement un sens de dire " (prêtant [...] attention à ce que je regarde [während des aufmerksamen Schauens]), je me représente [vorstellen] tout ce qu'on voudra ${ }^{1}$ : celui de la lecture d'un livre par exemple. Mais même dans un cas de ce type, les expériences sont concomittantes mais pas identiques : pendant la lecture, je regarde des mots et j'imagine des histoires.

- Deuxième remarque : Wittgenstein compare les énoncés « essayer de voir quelque chose» et " essayer de se représenter quelque chose». Il remarque que "dans le premier cas on dit quelque chose comme "Regarde bien!", dans le second: "Ferme les yeux!" $»^{2}$. Les deux jeux de langage semblent donc opérer différemment: l'un semble nécessairement lié à la perception externe, l'autre pas, du moins pas nécessairement.

- Troisième remarque : c'est un non-sens de dire que quelque chose pourrait «se présenter comme se présente par exemple l'impression visuelle que j'ai en ce moment, mais par ailleurs se comporter comme se comporte une représentation $»^{3}$. De même, comme le remarque le paragraphe 97, «il est dans ma nature de ne pouvoir prendre une impression pour une représentation » car il n'existe pas de «cas dans lequel quelqu'un d'autre le ferait $»{ }^{4}$. On ne peut donc pas substituer l'un à l'autre les concepts de représentation et d'impression car il n'est pas d'usage de le faire.

On pourrait multiplier les remarques de ce type à l'envi et c'est bien l'enjeu de l'analyse grammaticale menée dans les Remarques sur la philosophie de la psychologie. Nous retenons pour notre part, comme nous l'indique clairement Wittgenstein au paragraphe 75 des mêmes Remarques, qu'à la question de savoir si "voir et se représenter sont des phénomènes différents », il faut d'abord répondre que « les mots "voir" et "se représenter" ne sont pas employés de la même façon $»^{5}$. De l'analyse descriptive des jeux de langage qui mobilisent ces différents concepts, il ressort en effet qu'il y a des choses que l'on dit des impressions sensorielles ou des images visuelles que l'on ne dit pas des représentations. Certes, si l'on suit Wittgenstein, il est dangereux d'absolutiser ces différences dans la mesure où elles ne font sens que dans les contextes où elles sont appliquées. On peut cependant s'appuyer prudemment sur ces observations pour tenter d'identifier plusieurs critères susceptibles de distinguer ces différentes expériences en contexte.

1 RPPII $\S 65$, p. 14.

2 RPPII $\S 72$, p. 15.

3 RPPII $\S 95$, p. 21

4 RPPII § 97, p. 21.

5 RPP II $\S 75$ p. 16. 


\section{Quelques spécificités grammaticales de la représentation par imagination}

\subsection{Imagination et volonté}

Si l'on suit les analyses de Wittgenstein, un premier critère susceptible de distinguer la représentation de l'impression sensible semble se dégager. Pour reprendre son expression, il semble en effet que seule l'imagination soit « soumise à la volonté » ${ }^{1}$. Ainsi que le formule encore Wittgenstein : «Les représentations sont volontaires, les sensations ne le sont pas $»^{2}$. On peut certes envisager des contextes où la volonté s'exerce aussi sur ce que nous voyons. Comme à son habitude, par sensibilité aux variations et aux cas particuliers, Wittgenstein imagine du moins un contexte où cela aurait un sens de le dire :

$\S 96$. Nous faisons regarder quelqu'un, à travers un trou, dans une sorte de kaléidoscope où nous faisons bouger divers objets et figures; et cela se produit de telle manière - soit par hasard, soit à dessein — que ce mouvement est précisément celui que l'observateur voulait, de sorte qu'il s'imagine que ce qu'il voit obéit à sa volonté3.

Mais, dans ce scénario, l'observateur est manifestement victime d'une illusion : il «s'imagine » (à tort) que « ce qu'il voit obéit à sa volonté », alors que ce qu'il voit obéit bien plutôt aux manipulations de la tierce personne qui connait bien ses volontés. Aussi, si l'on excepte le cas assez problématique du kaléidoscope, il semble légitime de dire que la vision n'est pas assujettie à la volonté alors que l'imagination l'est.

Cette remarque ne va cependant pas de soi. Comme le remarque encore Wittgenstein, il serait par exemple tentant de rétorquer que «bien souvent, des représentations s'imposent à nous contre notre propre volonté, et qu'elles persistent malgré nos efforts pour les chasser $»^{4}$. Il reste cependant juste de dire que

quand nous nous représentons quelque chose, nous n'observons pas. Que les images se forment et s'en aillent, ce n'est pas quelque chose qui nous arrive.

1 RPP II $\S 63$, p. 14.

2 RPP II $\S 129$, p. 28.

3 RPP II § 96, p. 21.

4 RPP II $\S 86$, p. 18. 
Nous ne sommes pas surpris par ces images, nous ne disons pas : « Regarde,

là $! . . . »^{1}$.

Qui plus est, cela a un sens d'affirmer que nous sommes en mesure de chasser certaines images, alors qu'il n'est pas du tout évident que nous puissions décider de ne pas voir une image visuelle ou ressentir une impression sensorielle. Wittgenstein essaie cependant d'imaginer un exemple. « Si je regarde ma main puis que je la fais sortir du champ visuel, n'ai-je pas interrompu volontairement l'impression visuelle que j'en avais? ». Or, selon Wittgenstein, même dans ce cas-là, « on n'appelle pas cela "chasser l'image de la main" " ${ }^{2}$. Il serait probablement plus naturel de dire que l'on a vu la main puis que l'on a décidé de ne plus la voir.

Pour ces différentes raisons, il semble légitime de dire que seule la représentation est volontaire. Il ne s'agit cependant ni d'une remarque empirique $^{3}$ ni même d'une remarque psychologique ${ }^{4}$. Il ne faut par exemple pas en conclure que «la volonté serait une sorte de moteur auquel les représentations seraient reliées et qui pourrait les susciter, les mouvoir, les supprimer $»^{5}$. Ce que suggère plutôt Wittgenstein, c'est qu' « il y a du sens à donner pour consigne: "Représente-toi ceci", et tout aussi bien "Ne te représente pas ceci" " ${ }^{6}$. C'est bien une remarque grammaticale.

\subsection{L'imagination: une pratique normée}

Par ailleurs, il semble légitime de dire que « Se représenter quelque chose est comparable à une activité [Tätigkeit] $»^{7}$ : au «dessin ${ }^{8}$, au «calcul mental ${ }^{9}$ ou même à la «nage ${ }^{10}$. Pour prendre un exemple de Wittgenstein, si l'on me demande d'imaginer une mélodie, « je me la chante intérieurement $»^{11}$. Ce critère est étroitement connecté au précédent. C'est

1 RPP II $\S 88$, p. 19.

2 RPP II $\S 91$ p. 19.

3 RPP II $\S 90$.

4 RPP II $\S 107$.

5 RPP II $\S 78$, p. 17.

6 RPP II $\S 83$, p. 18.

7 RPP II $\S 88$, p. 19.

8 RPP II $\S 80$.

9 RPP II $\S 81$.

10 RPP II $\S 88$.

11 RPP II $\S 81$. 
parce que l'on considère que l'imagination est « soumise à la volonté », que l'on peut ordonner à quelqu'un de se représenter telle ou telle image ${ }^{1}$ et que cela a un sens de décrire cette expérience comme une activité. Mais une telle activité est-elle soumise aux mêmes règles que celles qui régulent toutes les autres pratiques? Pour le dire autrement, l'imagination est-elle une pratique normée, c'est-à-dire une pratique qui doit être pratiquée selon des règles dictées par les circonstances?

Une précision au préalable. Selon Wittgenstein, les images des représentations elles-mêmes, à la différence des impressions sensorielles, "ne nous apprennent rien sur le monde extérieur, ni vrai ni faux $»^{2}$. Il faut donc bien distinguer les représentations de l'imagination des « hallucinations » ou des « imaginations » au sens d' « élucubrations » (Einbildungen). Les images des représentations n'obéissent à aucun critère de correction et cela n'a pas de sens de les caractériser comme correctes ou incorrectes.

Pour autant, Wittgenstein soutient l'idée selon laquelle l'activité d'imagination elle-même est soumise à des règles de corrections : il y a notamment des choses qu'il est impossible d'imaginer et d'autres dont on peut se faire, à tort, une représentation. Dans les Recherches philosophiques, Wittgenstein nous dit par exemple qu'il existe des propositions dont « je ne peux pas m'imaginer le contraire $»^{3}$. Quand quelqu'un me dit qu'il est « seul à pouvoir savoir s'[il] ressent une douleur », on peut répondre, « je ne peux pas m'imaginer le contraire de cela ", à savoir : je ne peux pas m'imaginer que quelqu'un d'autre pourrait savoir ce qu'il ressent. Inversement, si je crois pouvoir me faire une représentation de ce que cet individu ressent, mon activité d'imagination est « incorrecte» ou plus exactement elle n'a pas de sens. Il convient de noter que la portée d'une telle remarque est encore grammaticale. Aucune contrainte empirique ou physiologique ne pèse en effet sur nos facultés d'imagination. Cela «ne signifie évidemment pas ici que la puissance de mon imagination ne me permet pas [d'imaginer le contraire] ${ }^{4}$. Pour le formuler dans les termes de Wittgenstein, une proposition comme « je ne peux pas m'imaginer le contraire » n'est donc pas « une proposition d'expérience » mais c'est encore "une proposition grammaticale » que Wittgenstein suggère de reformuler en ces termes : plutôt que de dire : «Je ne peux pas me représenter le contraire », il serait encore plus

\footnotetext{
1 RPP II $\S 125$.

2 RPP II $\S 63$, p. 14.

3 RP $§ 251$, p. 138.

4 Ibidem.
} 
correct de dire « Je ne peux pas me représenter ce que tu dis » ${ }^{1}$ quand tu dis que tu imagines savoir ce qu'il ressent. C'est un non-sens de dire que l'on imagine une telle chose car, jusqu'à preuve du contraire, il n'existe pas de contexte pertinent où cette proposition aurait un sens.

Étant donnés nos contextes d'usage, il semble donc que certaines pratiques, en l'occurrence certaines pratiques de représentation, soient exclues du langage. C'est à ce titre que l'on peut dire que la pratique de représentation est normée en fonction des attentes contextuelles: je ne peux pas imaginer n'importe quoi. Il faut encore que ce que j'imagine ait un sens (et donc un usage) dans le langage.

\section{L'imagination : un acte créateur}

Dernier critère grammatical que nous retenons : si l'imagination doit respecter certaines règles, il n'en demeure pas moins que l'imagination est une activité créatrice. Dans les Remarques sur la philosophie de la psychologie, Wittgenstein caractérise l'activité de représentation en ces termes :

Dire qu'il y a une différence entre image visuelle [Gesichtsbild] et image dans la représentation [Vorstellungsbild], cela signifie qu'on se représente quelque chose autrement qu'elle ne se montre ${ }^{2}$.

Pour prendre un exemple de Wittgenstein, si l'on demande à quelqu'un " Dessine Untel d'après la représentation que tu en as ", « ce n'est pas d'après la ressemblance du portrait que l'on décidera s'il l'a fait ou non $»^{3}$. Comme le disait déjà Hume (et sur ce point il existe une forme de continuité entre les deux penseurs), la représentation de l'imagination se distingue dès lors par son grand degré de liberté à l'égard des impressions sensorielles. Ainsi que le formule plus clairement encore Wittgenstein quelques lignes plus loin :

Le concept «se représenter » est plus proche du concept «agir» que du concept « recevoir ». "Se représenter » pourrait être appelé un acte créateur [ein schöpfericher Akt]. (Et de fait, il est aussi appelé de cette façon. $)^{4}$

\footnotetext{
1 Ibidem.

2 RPP II $\S 69$, p. 15.

3 RPP II $\S 82$, p. 17.

4 RPP II $\S 111$, p. 24.
} 
Plus qu'une pratique normée, c'est donc un acte créateur libéré des contraintes de ressemblance.

Il résulte de cette spécificité créatrice le fait que l'imagination se caractérise par une fécondité exceptionnelle et qu'elle joue à ce titre un rôle privilégié dans la méthodologie wittgensteinienne. Elle joue en effet un rôle fondamental dans l'économie de la vision synoptique que Wittgenstein appelle de ses vœux depuis son retour à Cambridge du début des années 1930. Comme le manifestent par exemple très clairement ses Dictées à Waismann et pour Schlick, Wittgenstein présente sa méthode comme une «présentation synoptique » (übersichtliche Darstellung) des différents jeux de langage, destinée à clarifier l'usage des concepts du langage. Cette méthode s'inscrit dans la filiation de la méthode goethéenne présentée dans la Métamorphose des plantes: de même que Goethe préconise d'analyser une plante dans son milieu, puis de la comparer à d'autres, la méthode philosophique que préconise Wittgenstein consiste à placer une forme linguistique dans son contexte et à la comparer, à l'aide de jeux de langage comparatifs, à d'autres formes linguistiques. Ainsi seulement, pour reprendre le terme utilisé également par Goethe, obtient-on une «vue de surface» ou «vue synoptique» (Übersicht) des plantes ou des concepts: une image d'ensemble. Or, le recours à l'imagination de «cas fictifs » est l'outil par excellence de cette méthode. Comme le formule Wittgenstein dans ses Dictées : "Nous construisons le cas fictif particulier d'un jeu, dans le but d'examiner ce qui se passe réellement sous le point de vue de l'analogie avec cet autre jeu $»^{2}$. Ainsi que le commente Denis Perrin dans un article de 2011, «La construction de cas fictifs est en effet un élément essentiel de la nouvelle présentation synoptique, qui trouve en eux les "objets de comparaison" au moyen desquels elle procède $»^{3}$. L'imagination de cas fictifs joue donc un rôle fondamental dans la logique de la méthode de comparaison à laquelle a systématiquement recours Wittgenstein dans sa dite deuxième philosophie : cette pratique est susceptible de procurer au philosophe de nouveaux objets de comparaison et de lui fournir une image d'ensemble de tous les cas particuliers.

1 Dictées de Wittgenstein à Waismann et pour Schlick. Vol. 1, G. Baker, B. McGuinness et A. Soulez (éds.), tr. fr. A. Soulez (dir.), Paris, PUF, 1997.

${ }^{2}$ Dictées..., op. cit., « Grammaire de la règle », p. 138.

3 D. Perrin, "L'intelligibilité synoptique. Ressemblance, aspect, morphologie», $L^{\prime}$ 'Art du comprendre. Wittgenstein et les questions du sens, $\mathrm{n}^{\circ} 20$ (2011), p. 17.

104 


\section{Conclusion}

Il ressort de cette analyse qu'il est possible d'identifier certaines différences entre les jeux de langage qui mobilisent les concepts de représentation par image et ceux d'impressions sensorielles. Par l'analyse des remarques grammaticales de Wittgenstein, nous avons en effet pu caractériser trois critères susceptibles de distinguer ces jeux de langage: 1/ la pratique d'imagination ou «représentation» est soumise à la volonté ; $2 /$ c'est une pratique, et en conséquence une pratique normée, au sein de l'institution du langage ; 3/ c'est une pratique libérée de tout assujettissement aux impressions sensorielles : un acte créateur.

À la lecture de ces différentes remarques de Wittgenstein, on comprend que le philosophe autrichien s'intéresse davantage, du moins en 1948, à la dimension pratique de l'imagination qu'à l'objet de l'imagination ou à l'image imaginée. Il est certes parfois question de l'image dans la représentation (Vorstellungsbild), par exemple au paragraphe 69 des Remarques sur la philosophie de la psychologie, mais l'analyse porte le plus souvent sur l'acte de représentation lui-même (Vorstellen) qui se caractérise par sa capacité de représentation de cas fictifs. Notons à titre d'argument que les différents cas imaginés n'ont pas nécessairement le format d'une image. Selon Wittgenstein, il est en effet tout à fait possible d' ' imaginer» un langage, une hypothèse, un usage, un contexte ou toute autre chose. Aussi, au sens strict, la représentation de la pratique imaginative n'est pas nécessairement une image. Plus précisément, comme le dit Wittgenstein dans les Recherches philosophiques, "Une représentation n'est pas une image, mais une image peut lui correspondre $»^{1}$. C'est la raison pour laquelle, chez Wittgenstein, on peut parler d'imagination sans image.

Cependant, Wittgenstein ne considère pas l'imagination comme une activité purement mentale. Il existe certes des différences significatives entre l'activité de représentation et la perception sensible, mais Wittgenstein insiste tout autant sur les parentés évidentes entre les jeux de langage qui mobilisent ces concepts :

Il n'y a rien de plus faux que de dire que voir [sehen] et se représenter [vorstellen] sont des activités différentes. C'est comme si l'on disait qu'aux échecs jouer un coup et perdre sont deux activités différentes ${ }^{2}$.

$1 \mathrm{RP} \S 301$, p. 152.

2 RPP II $\S 138$, p. 29. 
Certes, il existe des représentations sans image, mais, «à la question "que représentes-tu ?", on peut répondre par une image ${ }^{1}$.

En conclusion, on comprend qu'il est tout aussi contestable de considérer l'imagination comme une perception sensible d'impressions sensorielles que comme une activité de formation d'images mentales. Selon sa méthode habituelle, Wittgenstein nous invite bien plutôt à être sensibles aux variations et aux différentes articulations possibles entre les concepts d'image, de représentation et d'impression sensible. Il n'en reste pas moins que la pratique imaginative - entendue comme l'invention de cas fictifs s'avère un outil particulièrement précieux pour mettre en œuvre ce réquisit méthodologique et manifester ces différences conceptuelles.

\section{Bibliographie}

Baker G., McGuinness B. (éds.), Dictées de Wittgenstein à Waismann et pour Schlick. Vol. 1, tr. fr. A. Soulez (dir.), Paris, PUF, 1997.

Glock H.-G., A Wittgenstein Dictionary, Oxford, Blackwell, 1996.

Hume D., Traité de la nature humaine, tr. fr. Ph. Baranger et Ph. Saltel, Paris, Flammarion, 1995.

Husserl E., Recherche logique 1. T. II/vol. 1 : Recherches pour la phénoménologie et la théorie de la connaissance, Paris, PUF, 1961.

Perrin D., «L'intelligibilité synoptique. Ressemblance, aspect, morphologie », L'Art du comprendre. Wittgenstein et les questions du sens, $\mathrm{n}^{\circ} 20$ (2011).

Sartre J.-P., L'Imaginaire, Paris, Gallimard, 1940.

Wittgenstein L., Bemerkungen über die Grundlagen der Mathematik (1937-1944), G.E.M. Anscombe, G.H. von Wright et R. Rhees (éds.), Bd 6, Francfort-sur-leMain, Suhrkamp, 1954, trad. fr. M.-A. Lescourret, Remarques sur les fondements des mathématiques, Paris, Gallimard, 1983.

Wittgenstein L., Bemerkungen über die Philosophie der Psychologie II (1948), G.E.M. Anscombe et G.H von Wright (éds.), Oxford, Blackwell, 1980, tr. fr. G. Granel, Remarques sur la philosophie de la psychologie II (RPP II), Mauvezin, TER, 1994.

Wittgenstein L., Philosophische Untersuchungen/ Philosophical Investigations (Partie $1: 1945$; Partie $2: 1947-1949)$, G.E.M. Anscombe et R. Rhees (éds.), tr. fr. F. Dastur, M. Elie, J-L. Gautero, D. Janicaud, É. Rigal, Recherches philosophiques (RP), Paris, Gallimard, 2004.

1 RPP II $\S 63$, p. 14. 
Wittgenstein L., Zettel (1945-1948), G.E.M. Anscombe et G.H. von Wright (éds.), trad. angl. G.E.M. Anscombe, Oxford, Blackwell, 1967, tr. fr. J.-P. Cometti et É. Rigal, Fiches (F), Paris, Gallimard, 2008. 